\title{
Structure-preserving low-order modeling approach of laminated composite plates integrated with macro-fiber composite transducers for dynamic applications
}

\author{
ZhongZhe Dong ${ }^{\mathrm{a}, \mathrm{b}, \mathrm{c}, \text { *, Cassio Faria }}{ }^{\mathrm{d}}$, Bert Pluymers ${ }^{\mathrm{a}, \mathrm{b}}$, Martin Hromčík ${ }^{\mathrm{c}}$, Michael Šebek ${ }^{\mathrm{c}}$, Wim Desmet ${ }^{\mathrm{a}, \mathrm{b}}$ \\ ${ }^{a}$ KU Leuven, Department of Mechanical Engineering PMA, Celestijnenlaan 300B, 3001 Heverlee, Belgium \\ ${ }^{\mathrm{b}}$ DMMS Lab, Flanders Make, Belgium

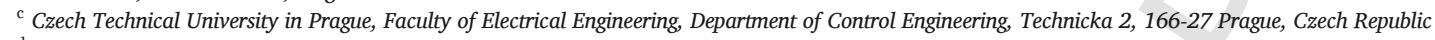 \\ ${ }^{\mathrm{d}}$ Siemens PLM Software, Engineering Service Division RTD, Interleuvenlaan 68, 3001 Leuven, Belgium
}

\section{ART ICLE INFO}

\section{Keywords:}

Composite laminated plate

Macro-fiber composite transducers

Structure-preserving

Low-order models

Dynamic applications

\begin{abstract}
A B S T R A C T
An Equivalent Substructure Modeling (ESM) approach of laminated composite plates with integrated macro-fiber composite (MFC) transducers is described. The proposed approach generates structure-preserving low-order system models for dynamic applications such as vibration suppression and energy harvesting. The direct piezoelectric effect of MFC transducers is derived from the electrical boundary conditions and it has the same coupling patterns as equivalent forces which describe the inverse piezoelectric effect. Hence, the reversibility of the piezoelectric effect is ensured and the electrical dynamics of the system can be simulated. The dual electromechanical couplings are assigned to a low-order structural model generated by an equivalent substructure concept. A laminated composite plate with integrated MFC transducers is used for validations and the simulated results agree well with experimental data. Two given study cases demonstrated that the ESM approach not only can generate accurate low-order system models but also provides a flexible fashion to design piezoelectric composite systems.
\end{abstract}

\section{Introduction}

Composite structures are increasingly used for a wide range of applications in engineering. The laminated composites with layers which could be isotropic or anisotropic provide numerous options for the design of lightweight mechanical components which retain high static stiffness and impact resistance. However, such kind of lightweight structures could be sensitive to external disturbances due to the high stiffness to mass ratio and result in noise and vibration issues. Composite structure consisting of mutlifunctional materials which are employed as sensors or actuators provide a promising potential to confront the challenges [1].

The hybrid piezoelectric composite, named Macro-fiber composite, is growingly used because of its high performance, mechanical flexibility and ease of integration [2,3]. They can be used for actuation to generate mechanical motions with an electric input (Inverse piezoelectric effect) and sensing to produce an electric output through mechanical deformations (direct piezoelectric effect). Therefore, there are many studies investigated vibration rejection and energy harvesting on composite structures by using MFC transducers [4-10].

A comprehensive design of composite structures with spatial distributed MFC transducers is essential to appropriately deploy the control systems in dynamic applications. Finite Element Modeling (FEM) methods are widely used to model piezoelectric structures $[6,11-14]$ because the dual electromechanical couplings can be simulated through strain field. The FEM methods can also solve complex structures including the presence of transducers. However, the FEM models commonly need to be reduced to a suitable size in order to be applicable in dynamic applications. The common model order reduction techniques such as Krylov subspace projection [15] and balanced truncation [16] modify the original system model so that a lot important physical information of the system such as the placement of the transducers and structural mode shapes is not preserved in the reduced-order model. An additional computing effort is required to update the system model if certain parameters of the integrated transducers should be revised to satisfy specific requirements. Hence, it is favorable to relax the dependency between host structures and the integrated MFC transducers in modeling. A semi-analytical modeling approach

\footnotetext{
* Corresponding author at: KU Leuven, Department of Mechanical Engineering PMA, Celestijnenlaan 300B, 3001 Heverlee, Belgium.

Email address: zhongzhe.dong@kuleuven.be (Z. Dong)
} 


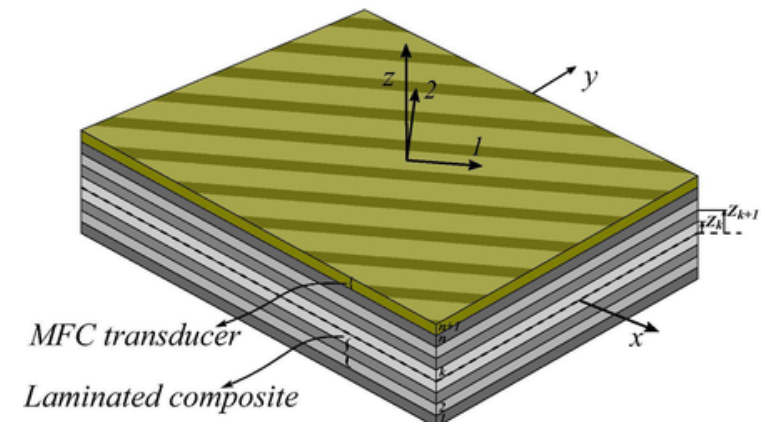

Fig. 1. Lay-up of laminated composite plate integrated with MFC transducer.

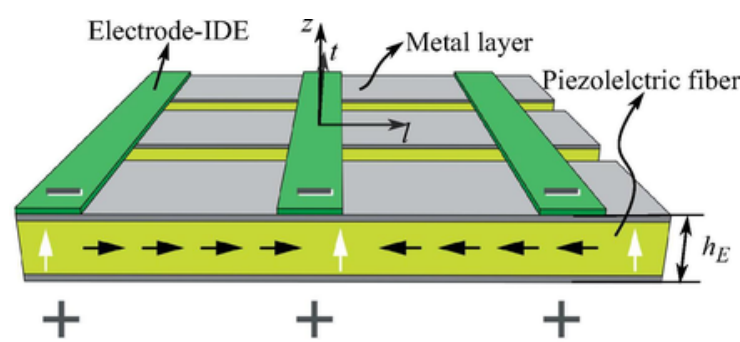

Fig. 2. Work principle of MFC-d31 transducers [28] (The white arrows represent the operational electric field and the black arrows indicate the deformation of the transducer.).

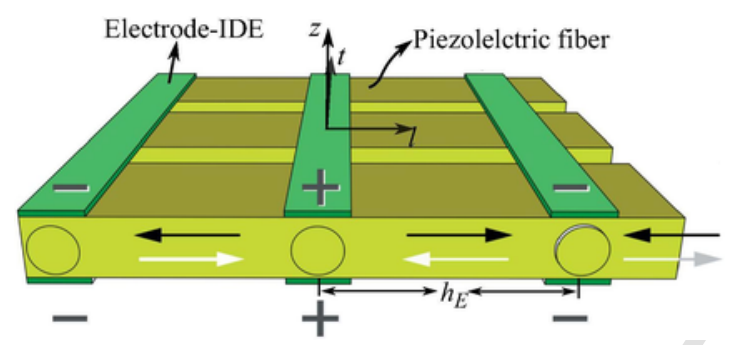

Fig. 3. Work principle of MFC-d33 transducers [28] (The white arrows represent the operational electric field and the black arrows indicate the deformation of the transducer.).

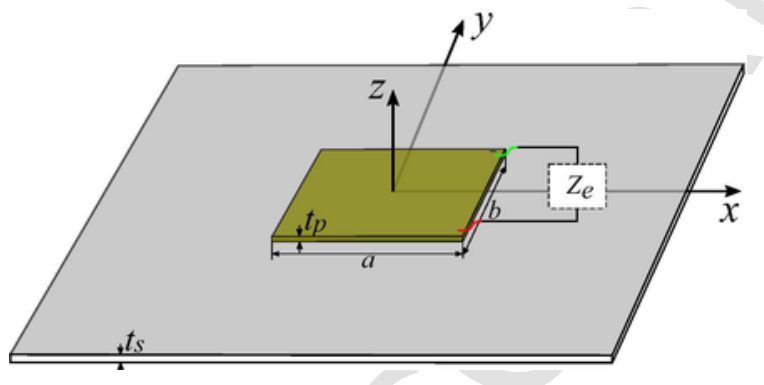

Fig. 4. A plate with integrated MFC transducer.

which decouples the mechanical and piezoelectric aspects of piezoelectric structures is useful because the inverse piezoelectric coupling can be individually assigned to mechanical models as equivalent loads. Early characterization of equivalent loads for anisotropic piezoelectric actuators has been performed in [17] by using spatial distributions. Later on, the equivalent loads has been generalized for anisotropic piezoelectric actuators with arbitrary shapes through both the Green functions and the Hamilton's principle in [18]. The equivalent loads in both work are expressed in the combination of force and bending moments. Recently, the equivalent loads in terms of force is reported in [19]. Hence, the inverse piezoelectric effect are well characterized by the equivalent loads but the corresponding direct piezoelectric coupling has not been discussed yet. So the piezoelectric induced effect by the transducers cannot be simulated in a semi-analytical modeling approach.

In this paper, the equivalent substructure modeling approach of laminated composite plates with integrated MFC transducers is presented. The direct piezoelectric effect of MFC transducer is derived and coupled with equivalent force to ensure the reversibility of piezoelectric phenomena. The equivalent substructure concept is used to generate low-order structural models. MFC transducers are considered as substructures that can be directly integrated on the equivalent plate model through nodal connections. The dual electromechanical couplings are transformed to the low-order structural model. Therefore, the composite plate and the integrated MFC transducers are separately treated in modeling and the structure of the system model is preserved. The low-order system model not only offers high flexibility to design piezoelectric transducer integrated structures but also directly serves to dynamic applications.

The paper is structured as follows: Firstly, the constitutive relations of a laminated composite plate with integrated rectangular MFC transducers are described in Section 2. And the analytical characterization of the MFC transducers is expressed in Section 3. The equivalent substructures modeling is presented in Section 4. Then, the dynamic validations of the proposed modeling approach are given in Section 5. Two study cases of energy harvesting and piezoelectric shunted damping are presented in Section 6 to demonstrate the capability of the presented modeling approach. Finally, conclusions are drawn in Section 7.

\section{Constitutive relations}

The lay-up of a laminated composite plate integrated with a MFC transducer is shown in Fig. 1. Each layer of the plate has transverse ortotropic properties. The plane stress assumption is adopted because the thickness of the plate is small comparing to the in-plane dimensions of the plate. The constitutive equations of the $k^{\text {th }}$ layer can be derived from Hooke's law in the material coordinates $(1,2)$ :

$\left\{\begin{array}{l}T_{x x} \\ T_{y y} \\ T_{x y}\end{array}\right\}=\left[\begin{array}{ccc}Q_{11} & Q_{12} & 0 \\ Q_{21} & Q_{22} & 0 \\ 0 & 0 & Q_{66}\end{array}\right]^{(k)}\left\{\begin{array}{l}S_{x x} \\ S_{y y} \\ S_{x y}\end{array}\right\}^{(k)}$

where

$$
\begin{aligned}
Q_{11} & =\frac{Y_{1}}{1-v_{12} v_{21}}, Q_{12} \\
& =\frac{v_{12} Y_{1}}{1-v_{12} v_{21}}, Q_{22} \\
& =\frac{Y_{2}}{1-v_{12} v_{21}}, Q_{21} \\
& =Q_{12}, Q_{66} \\
& =G_{12} .
\end{aligned}
$$

$T_{i j}, S_{i j}$ are the stress and strain components. $Y_{1}$ and $Y_{2}$ are the Young's moduli in the 1 and 2 directions in the material coordinates, respectively. $G_{12}$ is the shear moduli in 12 plane and $\nu_{i j}$ are the Poisson's ratios. Each layer of the laminated panel should be transformed into the global coordinates $(x, y)$ :

$\left\{\begin{array}{l}T_{x x} \\ T_{y y} \\ T_{x y}\end{array}\right\}=\left[\begin{array}{lll}\bar{Q}_{11} & \bar{Q}_{12} & \bar{Q}_{16} \\ \bar{Q}_{21} & \bar{Q}_{22} & \bar{Q}_{26} \\ \bar{Q}_{61} & \bar{Q}_{62} & \bar{Q}_{66}\end{array}\right]^{(k)}\left\{\begin{array}{l}S_{x x} \\ S_{y y} \\ S_{x y}\end{array}\right\}^{(k)}$

where $\bar{Q}_{i j}$ are the transformed material constants of the $k^{\text {th }}$ layer in the global coordinates.

With regard to the integrated MFC transducer, there are two types of MFC transducers: MFC-d33 and MFC-d31. The compositions of the two kinds of transducer are shown in Fig. 2 and 3, respectively. The MFC-d31 transducer uses the $e_{31}$ and $e_{32}$ effect that provide a compara- 


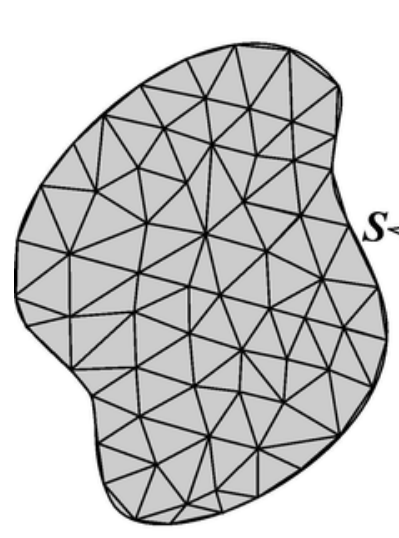

Orignal system

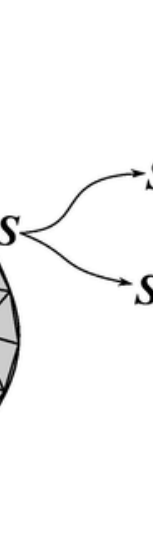

$-S_{2}$

Substructure decomposition

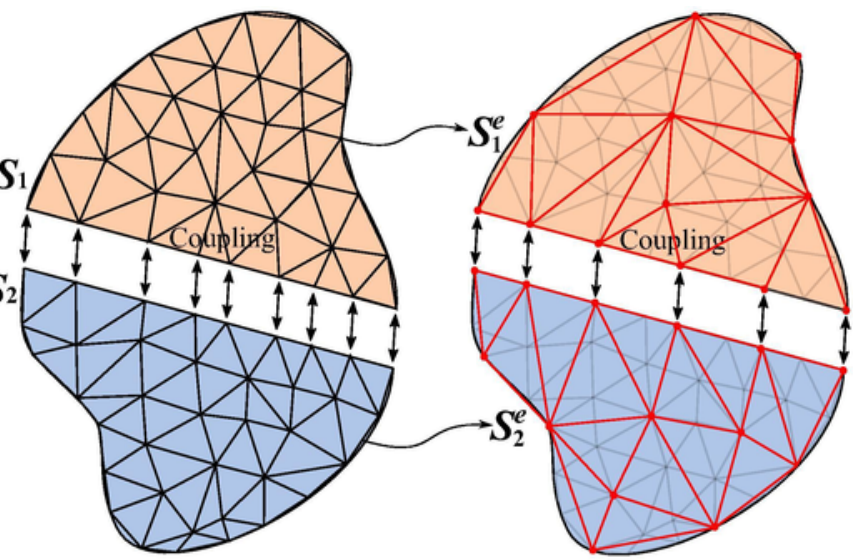

Equivalent system

Fig. 5. Equivalent substructure modeling concept.

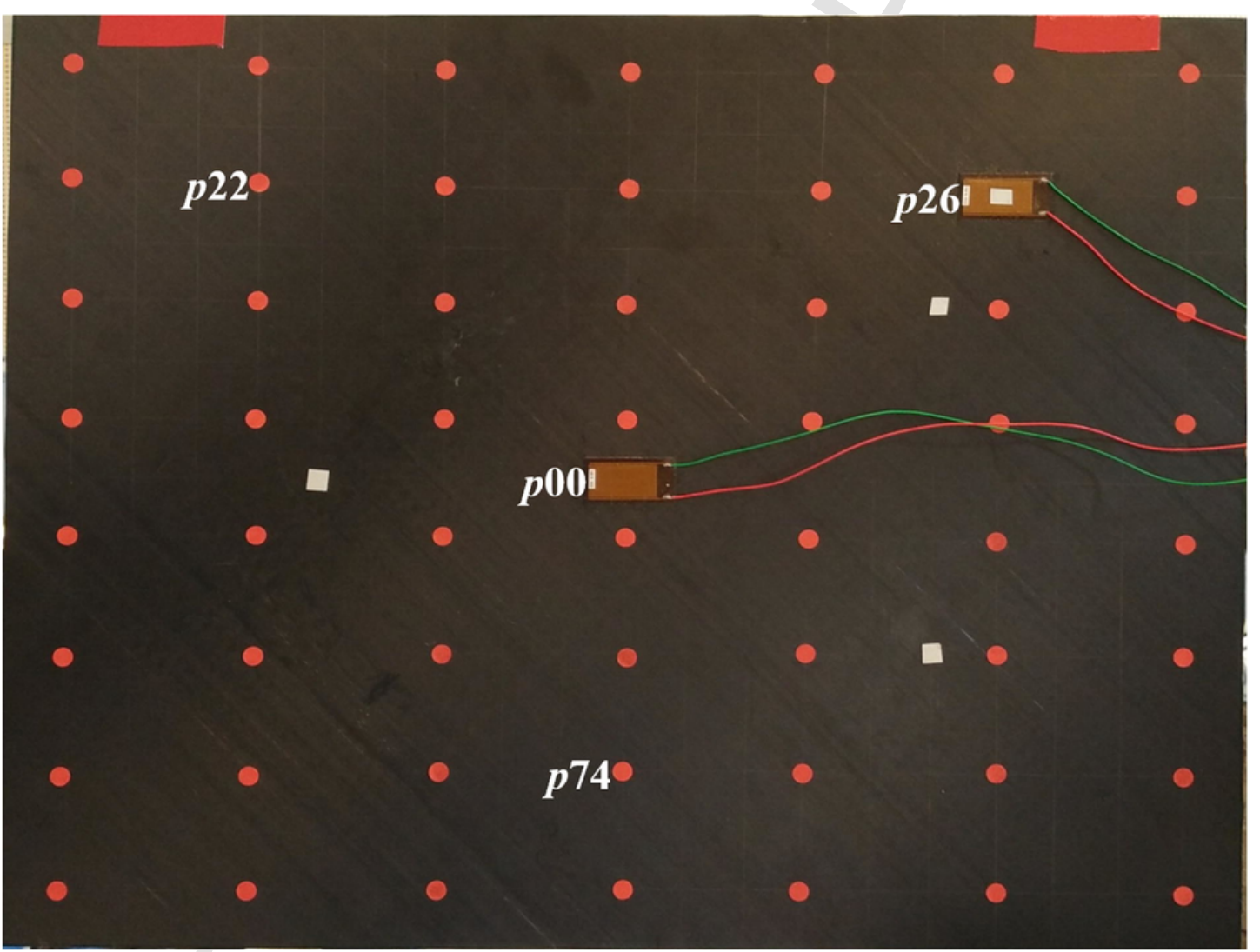

Fig. 6. Composite plate with integrated MFC transducers.

Table 1

Structure and material properties of the laminated composite plate with integrated MFC-d33 transducers.

\begin{tabular}{|c|c|c|c|c|c|c|c|c|c|}
\hline & Density $\left[\mathrm{Kg} / \mathrm{m}^{3}\right]$ & $c_{11}[\mathrm{GPa}]$ & $c_{22}[\mathrm{GPa}]$ & $G_{12}[\mathrm{GPa}]$ & $\nu_{12}$ & $G_{13}[\mathrm{GPa}]$ & $G_{23}[\mathrm{GPa}]$ & $d_{33}$ & $d_{32}$ \\
\hline MFC-d33 & 5440 & 27.142 & 14.8 & 4.1312 & 0.2922 & 5.385 & 10.5 & 272 & -133 \\
\hline Composite layer & 1500 & 110.15 & 7.868 & 4.05 & 0.26 & - & - & - & - \\
\hline
\end{tabular}

ble performance in both $x$ and $y$ directions. The operational electric field is applied to the thickness-wise direction of the transducer so that the electrode distance $h_{E}$ of the MFC-d31 equals to the thickness of the transducer - as shown in Fig. 2. The metal layer helps the electric field to be uniformly distributed on the piezoelectric fibers.
The interdigitated electrodes of MFC-d33 permit it to use the large $e_{33}$ effect. Then the electric field should be applied in the longitudinal direction of the piezoelectric fibers. The electrode distance is thus the distance between two electrodes' fingers as shown in Fig. 3. The operational electric field is not uniformly distributed overall the piezoelectric 


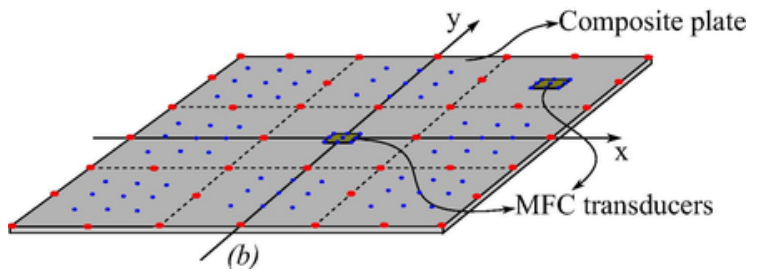

Fig. 7. ESM model of the composite plate with integrated MFC transducers (The red spots represent the border master nodes and the blue spots indicate the inner nodes on each substructure.) (For interpretation of the references to colour in this figure legend, the reader is referred to the web version of this article.).

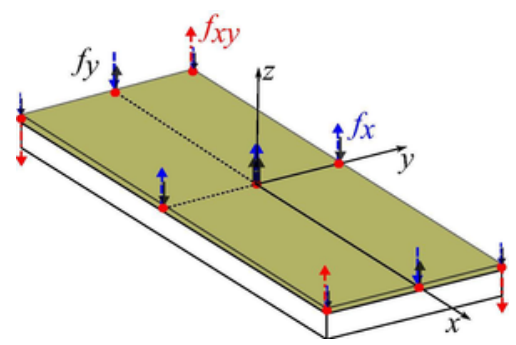

Fig. 8. Electromechanical couplings distribution on a rectangular MFC transducer $\left(f_{x}, f_{y}\right.$ and $f_{x y}$ represent the electromechanical coupling components of $\bar{e}_{31}, \bar{e}_{32}$ and $\bar{e}_{36}$ effects, respectively.)

fibers [20]. A convenient modeling approach can use the uniform electric field assumption which is corrected by a factor which is obtained based on the distribution shape of the operational electric field $[20,13]$.

The operational electric field should apply to $z$ direction on MFC-d31, but it applies to $x$ direction on MFC-d33. The distribution shapes of the electric field are also different. The different function mechanisms of the two types of transducer result in different piezoelectric constitutive equations in modeling [11]. However, the piezoelectric constitutive equations of MFC-d33 can be transformed into MFC-d31 form. The equivalent conversion must ensure that both the piezoelectric and electric properties of the MFC-d33 are retained [8]. Therefore, the following transformation can be performed:

$e_{31}^{\prime} \frac{V}{t_{p}}=e_{33} \frac{V}{h_{E}}, \quad e_{32}^{\prime} \frac{V}{t_{p}}=e_{32} \frac{V}{h_{E}}, \quad e_{36}^{\prime} \frac{V}{t_{p}}=e_{36} \frac{V}{h_{E}}$

where $h_{E}$ is the electrodes distance of MFC-d33 transducers and $t_{p}$ represents the electrode distance of MFC-d31 transducers. $V$ is the voltage. The dielectric constant needs also to be converted for remaining the capacitance of the MFC-d33 transducers. Then, the generalized two-dimensional piezoelectric constitutive equations based on in-plane stress assumption are introduced in the study for both the MFC-d31 and MFC-d33 transducers:

$\left\{\begin{array}{c}T_{x x} \\ T_{y y} \\ T_{x y} \\ D\end{array}\right\}=\left[\begin{array}{cccc}Q_{11}^{E} & Q_{12}^{E} & 0 & -e_{31} \\ Q_{21}^{E} & Q_{22}^{E} & 0 & -e_{32} \\ 0 & 0 & Q_{66}^{E} & -e_{36} \\ e_{31} & e_{32} & e_{36} & \epsilon^{s}\end{array}\right]\left\{\begin{array}{c}S_{x x} \\ S_{y y} \\ S_{x y} \\ E\end{array}\right\}$

where $E$ and $D$ are the electric field and electric displacement of the MFC transducers, respectively. $Q_{i j}^{E}$ are the Young's modulus under a given electric filed that defined in a similar way as given by (2). The
MFC-d33 transducer can be considered as a particular case of MFC-d31 transducer. $e_{31}$ denotes $e_{31}$ and $e_{31}^{\prime}$ for the MFC $-\mathrm{d} 31$ and $\mathrm{MFC}_{\text {indi- }}$ cates $e_{32}$ and $e_{32}^{\prime}$ for the MFC-d31 and MFC-d33 respectively; and $e_{36}$ expresses $e_{36}$ and $e_{36}^{\prime}$ for the MFC-d31 and MFC-d33 transducers respectively. $\left[\epsilon^{s}\right]$ is the corresponding dielectric constants. Similar to the orthotropic layer of the composite plate, the material properties of the piezoelectric layer need also to be transformed into the global coordinates $(x, y)$ :

$\left\{\begin{array}{c}T_{x x} \\ T_{y y} \\ T_{x y} \\ D\end{array}\right\}=\left[\begin{array}{cccc}\bar{Q}_{11}^{E} & \bar{Q}_{12}^{E} & \bar{Q}_{16}^{E} & -\bar{e}_{31} \\ \bar{Q}_{21}^{E} & \bar{Q}_{22}^{E} & \bar{Q}_{26}^{E} & -\bar{e}_{32} \\ \bar{Q}_{61}^{E} & \bar{Q}_{62}^{E} & \bar{Q}_{66}^{E} & -\bar{e}_{36} \\ \bar{e}_{31} & \bar{e}_{32} & \bar{e}_{36} & \epsilon^{s}\end{array}\right]\left\{\begin{array}{c}S_{x x} \\ S_{y y} \\ S_{x y} \\ E\end{array}\right\}$.

The mechanical contribution of the integrated MFC transducer can be considered an additional layer on the laminated composite plate as shown in Fig. 1.

\section{Piezoelectric modeling of rectangular MFC transducers}

As shown in Fig. 4, a rectangular MFC transducer of size $a \times b$ is integrated into a laminated composite plate. An electric circuit that may contain resistors, capacitors and inductors is connected to the transducer and its impedance is noted as $Z_{e}$. Let the structural coordinates of the transducer coincide with the global one on the plate for the convenience of analysis. Then the placement of the transducer can be expressed as follows in terms of the Heaviside functions $H$.

$$
\begin{aligned}
\Lambda_{x}^{p} \Lambda_{y}^{p}= & {\left[H\left(x+\frac{a}{2}\right)-H\left(x-\frac{a}{2}\right)\right] } \\
& \times\left[H\left(y+\frac{b}{2}\right)-H\left(y-\frac{b}{2}\right)\right] .
\end{aligned}
$$

According to (6), the piezoelectric effect of the transducer on the plate can be described as follows:

$D=\left[\bar{e}_{31} S_{x x}+2 \bar{e}_{36} S_{x y}+\bar{e}_{32} S_{y y}+\epsilon^{S} E\right] \Lambda_{x}^{p} \Lambda_{y}^{p}$.

Thus, the strain components depend on the placement of the transducer on the plate that is defined by the spatial distribution $\Lambda_{x}^{p} \Lambda_{y}^{p}$. The electric boundary condition of the piezoelectric system results in the following relation:

$\frac{d}{d t} \int_{A} D \times \vec{n} d A=\frac{V(t)}{Z_{e}}$

where $\vec{n}$ is the normal vector of the electrodes on the transducer, $D$ is the electric displacement, $V(t)$ is the voltage and $A$ is usually the overall surface of the electrodes. Substituting (8) into (9) yields the following generalized form on the plate:

$\frac{d}{d t} \int_{A_{s}}\left[\bar{e}_{31} S_{x x}+2 \bar{e}_{36} S_{x y}\right.$

$\left.+\bar{e}_{32} S_{y y}+\epsilon^{S} E\right] \Lambda_{x}^{p} \Lambda_{y}^{p} d A_{s}=\frac{V(t)}{Z_{e}}$

where $A_{s}$ is the surface area of the overall plate and $\Lambda_{x}^{p} \Lambda_{y}^{p}$ indicates the placement of the transducer on the plate. Given that the direct piezoelectric effect is desired to be coupled directly to the transverse displacement of the plate, the Kirchhoff strain-displacement relation has

Table 2

\begin{tabular}{|c|c|c|c|c|c|c|c|c|c|c|}
\hline Mode & $1^{s t}$ & $2^{n d}$ & $3^{r d}$ & $4^{\text {th }}$ & $5^{\text {th }}$ & $6^{\text {th }}$ & $7^{\text {th }}$ & $8^{\text {th }}$ & $9^{t h}$ & $10^{\text {th }}$ \\
\hline Experiment $[\mathrm{Hz}]$ & 39.8 & 48.3 & 77.0 & 109.2 & 113.3 & 150.9 & 196.3 & 198.1 & 215.2 & 259.2 \\
\hline FEM model $[\mathrm{Hz}]$ & 40.8 & 49.8 & 78.2 & 110.5 & 115.5 & 151.2 & 198.4 & 200.4 & 218.4 & 262.3 \\
\hline ESM model $[\mathrm{Hz}]$ & 41.6 & 49.6 & 76.6 & 110.3 & 116.0 & 149.3 & 195.8 & 199.0 & 216.5 & 257.4 \\
\hline
\end{tabular}

Natural frequencies comparison. 
Table 3

Coefficients of proportional damping.

\begin{tabular}{lll}
\hline & $\xi\left[\mathrm{s}^{-1}\right]$ & $\eta[\mathrm{s}]$ \\
\hline Value & 1.27 & $1.28 \times 10^{-6}$ \\
\hline
\end{tabular}

to be adopted in the analysis. The in-plane motion of the transducer is neglected on simple plate-type structures. (10) can be rewritten as:

$$
\begin{gathered}
\frac{d}{d t} \int_{Z_{p}} \int_{A_{s}}\left[-\bar{e}_{31} z \frac{\partial^{2} w(x, y, t)}{\partial x^{2}}-2 \bar{e}_{36} z \frac{\partial^{2} w(x, y, t)}{\partial x \partial y}-\bar{e}_{32} z \frac{\partial^{2} w(x, y, t)}{\partial y^{2}}\right. \\
\left.+\epsilon^{S} E\right] \Lambda_{x}^{p} \Lambda_{y}^{p} d A_{s} d z=\int_{Z_{p}} \frac{V(t)}{Z_{e}} d z
\end{gathered}
$$

where $Z_{p}$ indicates the thickness-wise dimension of the MFC transducer. Integrating (11) in the thickness-wise direction of the transducer yields:

$$
\begin{gathered}
\frac{d}{d t} \int_{A_{S}}\left[-\bar{e}_{31} \frac{z_{p}}{t_{p}} \frac{\partial^{2} w(x, y, t)}{\partial x^{2}}-2 \bar{e}_{36} \frac{z_{p}}{t_{p}} \frac{\partial^{2} w(x, y, t)}{\partial x \partial y}\right. \\
\left.-\bar{e}_{32} \frac{z_{p}}{t_{p}} \frac{\partial^{2} w(x, y, t)}{\partial y^{2}}+\epsilon^{S} E\right] \Lambda_{x}^{p} \Lambda_{y}^{p} d A_{s}=\frac{V(t)}{Z_{e}}
\end{gathered}
$$

in which,

$z_{p}=\int_{z_{S}}^{z_{S}+t_{p}} z d z$

The location of the transducer in the thickness-wise of the plate starts from $z_{s}$ to $z_{s}+t_{p}$ in the thickness-wise of the piezoelectric structure. The dielectric constant term in (12) can be expressed as follows with the consideration of $E=-V(t) / h_{E}$ :

$\int_{A_{S}} \epsilon^{S} E \Lambda_{x}^{p} \Lambda_{y}^{p} d A_{s}=-A_{p} \epsilon^{S} \frac{V(t)}{h_{E}}=-C_{p} V(t)$

where $A_{p}, h_{E}$ and $C_{p}$ are the surface area, electrodes distance and capacitance of the transducer, respectively. Substituting (14) into (12) yields:

$C_{p} \frac{\partial V(t)}{\partial t}+\frac{V(t)}{Z_{e}}=\int_{A_{s}}-\frac{z_{p}}{t_{p}}\left[\bar{e}_{31} \frac{\partial^{3} w(x, y, t)}{\partial t \partial x^{2}}+2 \bar{e}_{36} \frac{\partial^{3} w(x, y, t)}{\partial t \partial x \partial y}+\bar{e}_{32} \frac{\partial^{3} w(x,}{\partial t \partial y^{\prime}}\right.$

Substituting the spatial distribution of a rectangular MFC transducer of size $a \times b$ into (15) yields:

$$
\begin{gathered}
\left(C p \dot{V}(t)+\frac{V(t)}{Z_{e}}\right)= \\
-\frac{z_{p}}{t_{p}}\left[\bar{e}_{31} \int_{A_{s}} \frac{\partial^{2} \dot{w}(x, y, t)}{\partial x^{2}}\left[H\left(x+\frac{a}{2}\right)-H\left(x-\frac{a}{2}\right)\right] \times\left[H\left(y+\frac{b}{2}\right)-H\left(y-\frac{b}{2}\right)\right] .\right. \\
+\bar{e}_{32} \int_{A_{s}} \frac{\partial^{2} \dot{w}(x, y, t)}{\partial y^{2}}\left[H\left(x+\frac{a}{2}\right)-H\left(x-\frac{a}{2}\right)\right] \times\left[H\left(y+\frac{b}{2}\right)-H\left(y-\frac{b}{2}\right)\right] d A \\
+2 \bar{e}_{36} \int_{A_{s}} \frac{\partial^{2} \dot{w}(x, y, t)}{\partial x \partial y}\left[H\left(x+\frac{a}{2}\right)-H\left(x-\frac{a}{2}\right)\right] \times\left[H\left(y+\frac{b}{2}\right)-H\left(y-\frac{b}{2}\right)\right] d A
\end{gathered}
$$

where $\dot{\square}$ denotes the partial derivative respect to time $t$. The partial derivative terms respect to $x$ and $y$ in (16) are treated individually by integration by parts. (16) can be rewritten as:

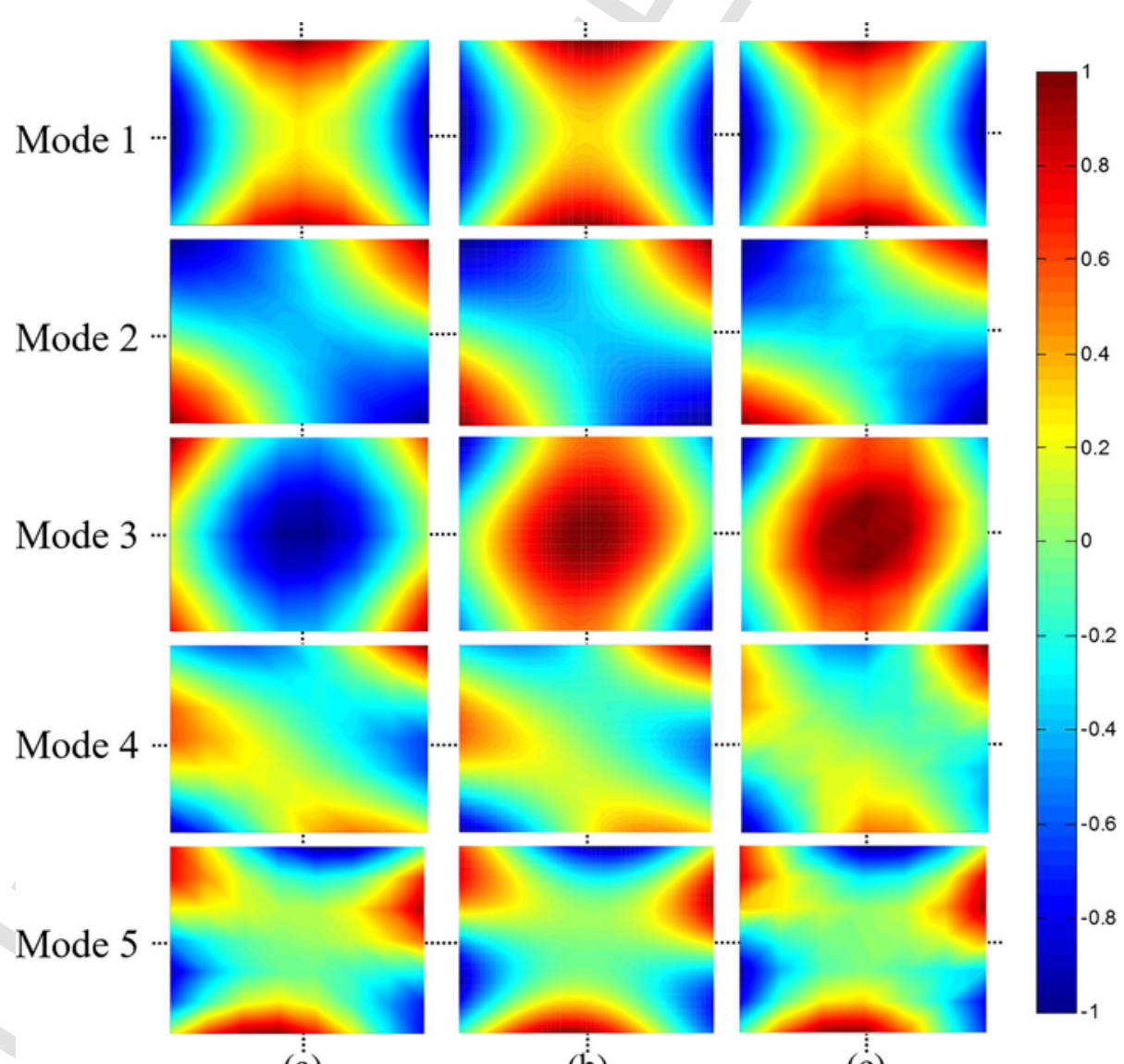

(a)

(b)

(c)

Fig. 9. Mode shapes validation: (a) Experimental data (b) FEM model, (c) ESM model. 

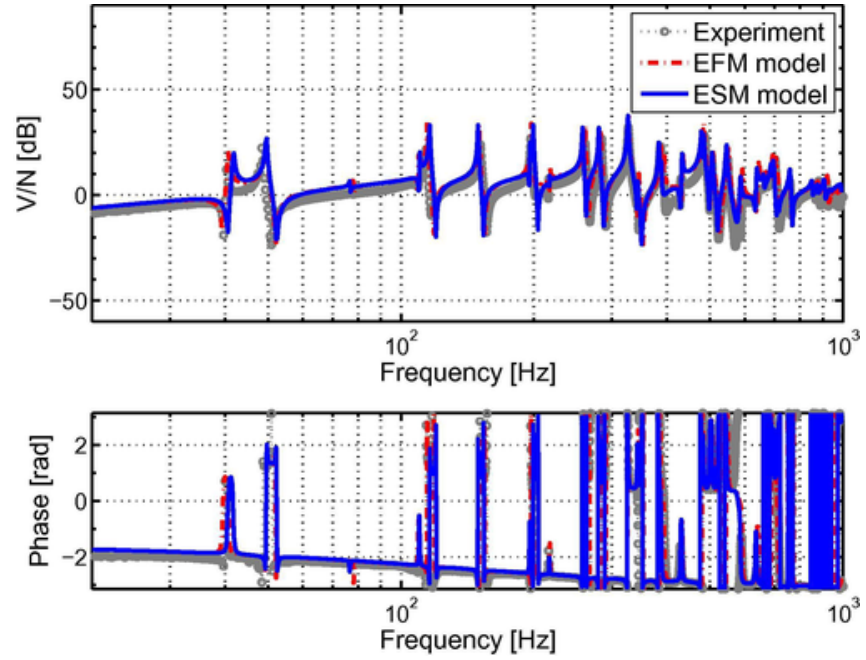

Fig. 10. Direct piezoelectric effect validation of the numerical models: voltage over force FRF p26-p26.
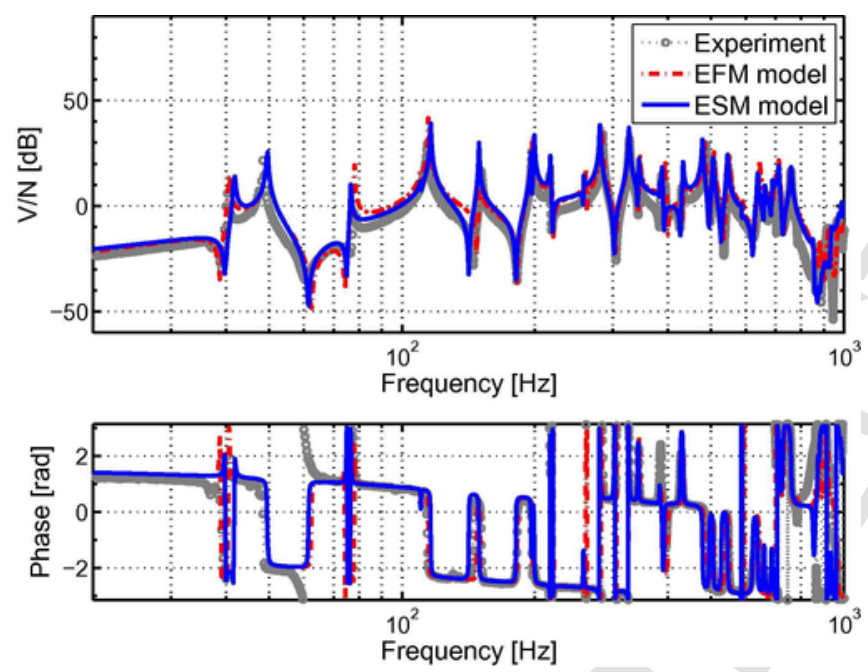

Fig. 11. Direct piezoelectric effect validation of the numerical models: voltage over force FRF p26-p22.

$$
\begin{gathered}
\left(C p \dot{V}(t)+\frac{V(t)}{Z_{e}}\right)= \\
-\frac{z_{p}}{t_{p}}\left[\bar{e}_{31} \int_{Y_{S}}\left(\left.\frac{\partial \dot{w}(x, y, t)}{\partial x}\right|_{x=\frac{a}{2}}-\left.\frac{\partial \dot{w}(x, y, t)}{\partial x}\right|_{x=-\frac{a}{2}}\right)\left[H\left(y+\frac{b}{2}\right)-H\left(y-\frac{b}{2}\right)\right] d y\right. \\
+\bar{e}_{32} \int_{X_{S}}\left(\left.\frac{\partial \dot{w}(x, y, t)}{\partial y}\right|_{y=\frac{b}{2}}-\left.\frac{\partial \dot{w}(x, y, t)}{\partial y}\right|_{y=-\frac{b}{2}}\right)\left[H\left(x+\frac{a}{2}\right)-H\left(x-\frac{a}{2}\right)\right] d x \\
\left.+2 \bar{e}_{36} \int_{A_{S}} \dot{w}(x, y, t)\left[\delta\left(x+\frac{a}{2}\right)-\delta\left(x-\frac{a}{2}\right)\right] \times\left[\delta\left(y+\frac{b}{2}\right)-\delta-\frac{b}{2}\right)\right] d A_{s}
\end{gathered}
$$

in which $X_{s}$ and $Y_{s}$ represent the dimensions of the composite plate in $x$ and $y$ directions, respectively. The first-order partial derivative terms in (17) can be approximated by first-order finite difference approach with uniform interval $d_{x}$ and $d_{y}$ in $x$ and $y$ directions, respectively. After that, the sampling property of the Dirac-delta function can be adopted to describe the finite difference terms. (17) is rewritten as follows:

$$
\begin{gathered}
\left(C p \dot{V}(t)+\frac{V(t)}{Z_{e}}\right) \\
=-\frac{z_{p}}{t_{p}} \bar{e}_{31} \frac{1}{d_{x}} \int_{A_{s}}\left[\delta\left(x-\frac{a}{2}\right)-\delta\left(x-\frac{a}{2}+d_{x}\right)-\delta\left(x+\frac{a}{2}-d_{x}\right)+\delta\left(x+\frac{a}{2}\right)\right] \\
\times\left[H\left(y+\frac{b}{2}\right)-H\left(y-\frac{b}{2}\right)\right] \dot{w}(x, y, t) d A_{s} \\
-\frac{z_{p}}{t_{p}} \bar{e}_{32} \frac{1}{d_{y}} \int_{A_{s}}\left[\delta\left(y-\frac{b}{2}\right)-\delta\left(y-\frac{b}{2}+d_{y}\right)-\delta\left(y+\frac{b}{2}-d_{y}\right)+\delta\left(y+\frac{b}{2}\right)\right] \\
\times\left[H\left(x+\frac{a}{2}\right)-H\left(x-\frac{a}{2}\right)\right] \dot{w}(x, y, t) d A_{s} \\
-2 \frac{z_{p}}{t_{p}} \bar{e}_{36} \int_{A_{s}}\left[\delta\left(x+\frac{a}{2}\right)-\delta\left(x-\frac{a}{2}\right)\right] \times\left[\delta\left(y+\frac{b}{2}\right)-\delta\left(y-\frac{b}{2}\right)\right] \dot{w}(x, y, t) d A
\end{gathered}
$$

One can observe that (18) has the same spatial distribution as the following equivalent force per unit area in (19) form [19] but with a negative sign $\left(t_{p} \equiv h_{E}\right)$. The same spatial distribution ensured the reversibility of the piezoelectric effect.

$$
\begin{aligned}
& p=\frac{V}{h_{E}} \bar{e}_{31} z_{p} \frac{\alpha_{x}}{d_{x}}\left[\delta\left(x+\frac{a}{2}\right)-\delta\left(x+\frac{a}{2}-d_{x}\right)-\delta\left(x-\frac{a}{2}+d_{x}\right)+\delta\left(x-\frac{a}{2}\right)\right. \\
& \times\left[H\left(y+\frac{b}{2}\right)-H\left(y-\frac{b}{2}\right)\right] \\
& +\frac{V}{h_{E}} \bar{e}_{32} z_{p} \frac{\alpha_{y}}{d_{y}}\left[\delta\left(y+\frac{b}{2}\right)-\delta\left(y+\frac{b}{2}-d_{y}\right)-\delta\left(y-\frac{b}{2}+d_{y}\right)+\delta\left(y-\frac{b}{2}\right.\right. \\
& \times\left[H\left(x+\frac{a}{2}\right)-H\left(x-\frac{a}{2}\right)\right] \\
& +2 \frac{V}{h_{E}} \bar{e}_{36} z_{p}\left[\delta\left(x+\frac{a}{2}\right)-\delta\left(x-\frac{a}{2}\right)\right] \times\left[\delta\left(y+\frac{b}{2}\right)-\delta\left(y-\frac{b}{2}\right)\right]
\end{aligned}
$$

where $\alpha_{x}=\frac{a}{a-d_{x}}$ and $\alpha_{y}=\frac{b}{b-d_{y}}$ are bending effort corrections along $x$ and $y$ directions, respectively [19]. It is important to mention that the finite difference approximation in (18) results in approximation errors too. $\alpha_{x}$ and $\alpha_{y}$ are applicable to compensate these errors because of the linear relation between bending moment and strain. The electromechanical couplings of MFC-d33 transducers can be obtained by introducing the (4) into the analysis. Finally, the dual electromechanical couplings in (18) and (19) can be rewritten as follows:

$\left(C_{p} \dot{V}+\frac{V}{Z_{e}}\right)=\widetilde{\boldsymbol{\Theta}}_{\phi w} \dot{\boldsymbol{w}}$

$p=\widetilde{\boldsymbol{\Theta}}_{w \phi} V$

where $\widetilde{\boldsymbol{\Theta}}_{\phi w}$ and $\widetilde{\boldsymbol{\Theta}}_{w \phi}$ are the direct and inverse piezoelectric coupling matrix of the transducers defined by (18) and (19), respectively. $\widetilde{\mathbf{\Theta}}_{\phi w}=-\widetilde{\boldsymbol{\Theta}}_{w \phi}^{T}$ where $\square^{T}$ denotes matrix transposition.$\phi$ indicates the electric degrees of freedom. It is noteworthy to mention that only the bending behavior of the structure which dominates the piezoelectric phenomena of the integrated MFC transducers is considered in the electromechanical couplings here. A uniform electric field assumption is adopted in (19), but the effective electric field on MFC-d33 transducers is not uniform due to the interdigitated electrodes. Therefore, an electric filed correction factor $\alpha_{E}$ defined in [20] should be introduced in the modeling of MFC-d33 transducer. Meanwhile, a shear-lag effect correction factor $\alpha_{p}$ due to the adhesive layer should be also included in experimental validations [21].

\section{Equivalent substructure modeling of composite plate integrated with MFC transducers}

In light with the work in [19], a FEM method is applicable to model the mechanical dynamics of the system. (20a) and (20b) can be assigned to the FEM model to simulate the dynamics of piezoelectric systems. But model order reduction is always required by the dynamic applications. Therefore, the equivalent substructure concept is introduced here to generate structure-preserving low-order system models. 

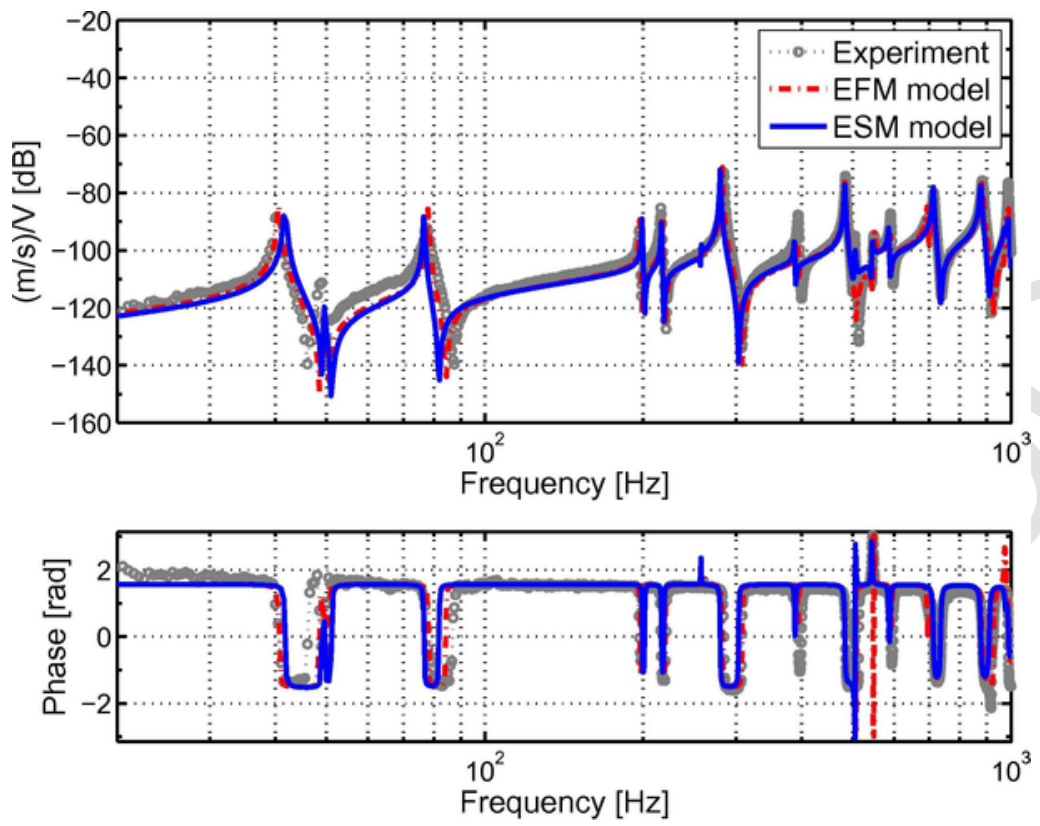

Fig. 12. Inverse piezoelectric effect validation of the numerical models: velocity over voltage FRF p00-p00.
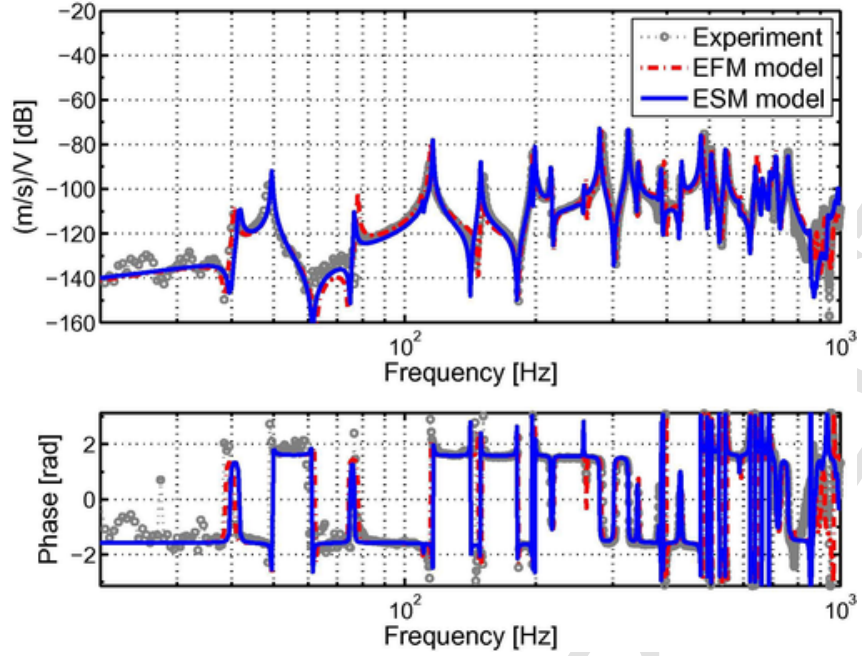

Fig. 13. Inverse piezoelectric effect validation of the numerical models: velocity over voltage FRF p26-p22.

As shown in Fig. 5, a mechanical structure $S$ can be divided into substructures. The dynamics of $S$ can be obtained by assembling the dynamics of the substructures $S_{1}$ and $S_{2}$. The idea of the equivalent substructures is to reduce the model of each substructure while the overall structural complexity is always retained after the assembly of $\boldsymbol{S}_{1}^{\boldsymbol{e}}$ and $\boldsymbol{S}_{2}^{e}$. According to the idea of a semi-analytical modeling approach, modeling of piezoelectric systems can be divided into mechanical and piezoelectric aspects. The mechanical dynamics of each substructure in free vibration can be described as follows:

$\widetilde{\boldsymbol{M}} \ddot{\mathbf{w}}+\widetilde{\boldsymbol{K}} \boldsymbol{w}=\mathbf{0}$

where $\tilde{\boldsymbol{M}}$ and $\tilde{\boldsymbol{K}}$ are the mass and stiffness matrix of a substructure generated by FEM methods, respectively. (21) can be partitioned according to the retained degrees of freedoms on master nodes subscripted by $r$ and deleted degrees of freedom on servant nodes subscripted by $d$ :

$\left[\begin{array}{ll}\tilde{\boldsymbol{M}}_{r r} & \widetilde{\boldsymbol{M}}_{r d} \\ \widetilde{\boldsymbol{M}}_{d r} & \widetilde{\boldsymbol{M}}_{d d}\end{array}\right]\left\{\begin{array}{l}\ddot{\boldsymbol{w}}_{r} \\ \ddot{\mathbf{w}}_{d}\end{array}\right\}+\left[\begin{array}{ll}\widetilde{\boldsymbol{K}}_{r r} & \widetilde{\boldsymbol{K}}_{r d} \\ \widetilde{\boldsymbol{K}}_{d r} & \widetilde{\boldsymbol{K}}_{d d}\end{array}\right]\left\{\begin{array}{c}\boldsymbol{w}_{r} \\ \boldsymbol{w}_{d}\end{array}\right\}=\left\{\begin{array}{l}\mathbf{0} \\ \mathbf{0}\end{array}\right\}$.

The second row in (22) leads to the following relation according to the static condensation:

$\boldsymbol{w}_{d}=\boldsymbol{R}^{(0)} \boldsymbol{w}_{r}$

where $\boldsymbol{R}^{(0)}=-\tilde{\boldsymbol{K}}_{d d}^{-1} \tilde{\boldsymbol{K}}_{d r}$. The reduced-order model of each substructure is obtained by introducing (23) into the first row in (22). The dynamic condensation is an iterative approach that allows the mass contribution can be approximately included in the order reduction transformation matrix. The new $R^{(i)}$ to replace $R^{(0)}$ in (23) is given as follows [22]:

Fig. 14. Voltage over force FRFs of the center MFC transducer for a set of piezoelectric fibrous orientations (The gray curve is experimental data.). 


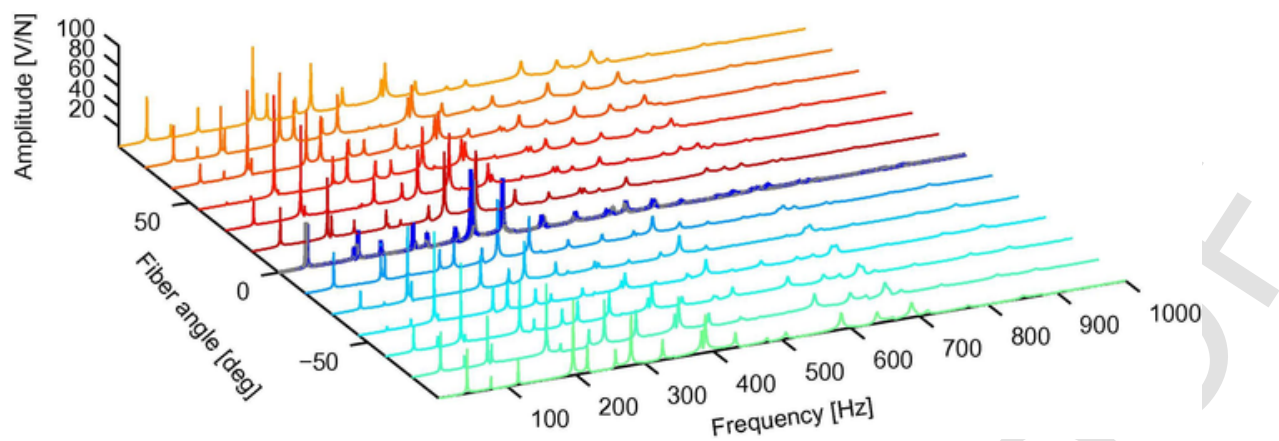

Fig. 15. Voltage over force FRFs of the corner MFC transducer for a set of piezoelectric fibrous orientations (The gray curve is experimental data.).

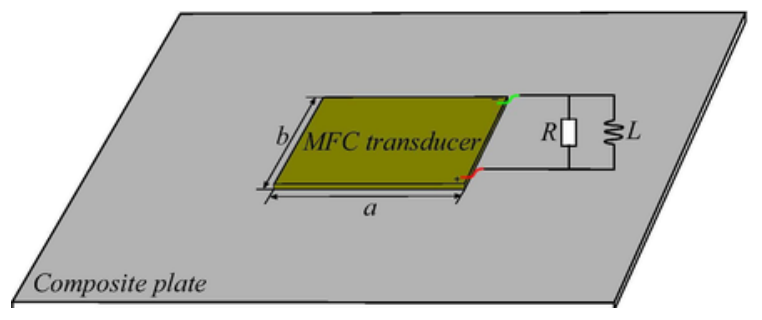

Fig. 16. Piezoelectric shunted damping system.
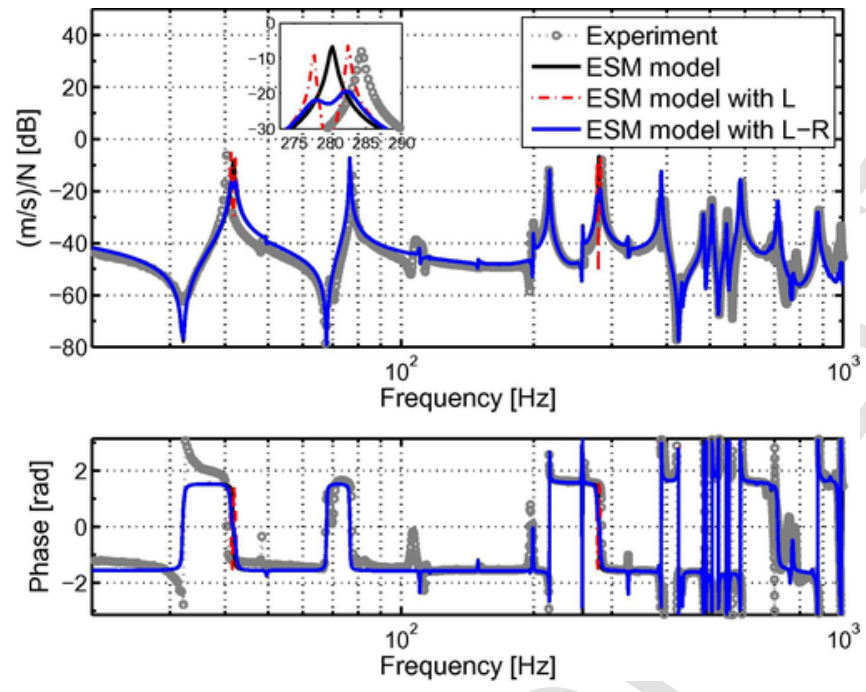

Fig. 17. Piezoelectric shunted damping on the composite plate: FRF p74-p00.

$$
\begin{array}{r}
\boldsymbol{R}^{(i)}=\tilde{\boldsymbol{K}}_{d d}^{-1}\left[\left(\tilde{\boldsymbol{M}}_{d r}+\tilde{\boldsymbol{M}}_{d d} \boldsymbol{R}^{(i-1)}\right)\left(\tilde{\boldsymbol{M}}_{s}^{(i-1)}\right)^{-1} \tilde{\boldsymbol{K}}_{s}^{(i-1)}\right. \\
\left.-\tilde{\boldsymbol{K}}_{d r}\right]
\end{array}
$$

where $\tilde{\boldsymbol{M}}_{s}^{(i-1)}$ and $\tilde{\boldsymbol{K}}_{s}^{(i-1)}$ are the reduced-order mass and stiffness matrix of each substructure from $i-1$ step $(i=1,2,3 \ldots)$. Therefore, the final order reduction transformation matrix is obtained:

$\boldsymbol{T}=\left[\begin{array}{c}\boldsymbol{I} \\ \boldsymbol{R}^{(i)}\end{array}\right]$

in which, $\boldsymbol{I}$ is an identity matrix. The reduced-order model of the substructure is thus expressed as:

$\bar{M} \ddot{\mathbf{w}}+\bar{K} \boldsymbol{w}=\mathbf{0}$. where $\overline{\boldsymbol{M}}=\boldsymbol{T}^{T} \tilde{\boldsymbol{M}} \boldsymbol{T}$ and $\overline{\boldsymbol{K}}=\boldsymbol{T}^{T} \tilde{\boldsymbol{K}} \boldsymbol{T}$ are the mass and stiffness matrix of the equivalent substructure model. When a MFC transducer is integrated into the plate substructure, the transducer equivalent substructure model can be obtained by following the above dynamic condensation technique. Meanwhile, the electromechanical couplings should be transformed to the equivalent substructure models. Let us take inverse piezoelectric coupling $\widetilde{\boldsymbol{\Theta}}_{w \phi}$ as an example. One can observe from the constitutive relations that the couplings are applied to the plate which dominates the mechanical dynamics of the system. Hence, the following transformation can be achieved:

$\widehat{\boldsymbol{\Theta}}_{w \phi}=\boldsymbol{T}^{T} \widetilde{\boldsymbol{\Theta}}_{w \phi}$

where $\boldsymbol{T}$ is the order reduction transformation matrix of the plate substructure model to which the transducers will be coupled. $\widehat{\boldsymbol{\Theta}}_{w \phi}$ is the transformed coupling matrix which is applied to all the master nodes of the plate equivalent substructure model. Now, a static condensation can be applied to $\widehat{\boldsymbol{\Theta}}_{w \phi}$ in order to converts the inverse piezoelectric coupling on the degrees of freedom which are used to couple the transducer:

$\overline{\boldsymbol{\Theta}}_{w \phi}=\boldsymbol{T}_{s}^{T} \widehat{\boldsymbol{\Theta}}_{w \phi}, \quad \boldsymbol{T}_{s}=\left[\begin{array}{c}\boldsymbol{I} \\ -\overline{\mathbf{K}}_{r d} \overline{\boldsymbol{K}}_{d d}^{-1}\end{array}\right]$

where $\overline{\boldsymbol{K}}$ is partitioned by the desired degrees of freedoms subscripted by $r$ and the rest of degrees of freedom subscripted by $d$. Similarly, the direct piezoelectric coupling can be transformed to the equivalent substructure models as follows:

$\overline{\boldsymbol{\Theta}}_{\phi w}=\widetilde{\boldsymbol{\Theta}}_{\phi w} \boldsymbol{T} \boldsymbol{T}_{s}$.

It is worthwhile to mention that the mass and stiffness influences of the transducer can be included into $\bar{M}$ and $\bar{K}$ before generates $\overline{\boldsymbol{\Theta}}_{w \phi}$ and $\overline{\boldsymbol{\Theta}}_{\phi w}$. Then, the presence of the transducer can be partially taken into account. This is also favorable for the assembling of the equivalent substructure models because the plate equivalent substructure models may be similarly constructed. Finally, the dynamics of the overall system including the electric circuit can be expressed as:

$\boldsymbol{M} \ddot{\mathbf{w}}+\boldsymbol{C} \dot{\mathbf{w}}+\boldsymbol{K} \boldsymbol{w}=\boldsymbol{F}+\boldsymbol{\Theta}_{w \phi} V$,

$C_{p} \dot{V}+\frac{V}{Z e}+\boldsymbol{\Theta}_{\phi w} \dot{\boldsymbol{w}}=0$

where $\boldsymbol{M}, \boldsymbol{C}$ and $\boldsymbol{K}$ are the mass, damping and stiffness matrix of the low-order structural model of the system, respectively. The equivalent force is used in the study but other equivalent loads are applicable to this approach too. Furthermore, it is possible to directly assign the electromechanical couplings $\widetilde{\boldsymbol{\Theta}}_{w \phi}$ and $\widetilde{\boldsymbol{\Theta}}_{\phi w}$ on the low-order structural models. The distribution on master nodes results in local coupling concentration and may leads to large error when the size of MFC transducers are considerable large that the wave length of the host plate modes. 


\section{Dynamic validation of ESM approach by a composite plate with integrated MFC-d33 transducers}

The experimental setup of the laminated composite plate with two integrated M2814P1 MFC-d33 transducers from [19] is used to validate the presented modeling approach. The laminated composite plate in Fig. 6 is made of CM-Perg TC-300 unidirectional layers with a symmetric lay-up $\left[\begin{array}{lll}-45^{\circ} 45^{\circ} & \circ 90^{\circ}\end{array}\right]_{S}$. The dimensions of the plate and the MFC transducer are $503 \mathrm{~mm} \times 400 \mathrm{~mm} \times 2.5 \mathrm{~mm}$ and $28 \mathrm{~mm} \times 14 \mathrm{~mm} \times 0.3 \mathrm{~mm}$, respectively. Both the MFC transducer actuation and sensing experimental data is used to validate the ESM approach. The material properties of the composite plate and MFC transducers are given in Table 1 [23]. The piezoelectric constants in the table are obtained form [24] for an $250 \mathrm{~V}$ peak to peak operational voltage with 0 offset.

As shown in Fig. 7, the plate is decomposed into 11 substructures in the ESM model. The unified approach for shear locking free triangular shell finite elements is used to model the structural model of each substructure [25]. Only 17 nodes are retained as master nodes on each plate substructure model. The master nodes on the plate substructure are classified into two groups: the inner nodes serving for the placement of transducers and sensors and the border nodes being used to couple the adjacent substructures. Meanwhile, the FE model of the transducer is reduced to a 9 nodes equivalent substructure model which is compatible with the inner nodes of the plate equivalent substructure model.

The corresponding distribution of the two electromechanical couplings on the master modes of MFC-d33 transducers is shown in Fig. 8. It is worthwhile to note that the master nodes configuration of each substructure is not unique. It is important to select the optimal configuration for each substructure if the electromechanical coupling is directly assigned on the equivalent substructure models so that the fidelity of the system model can be ensured.

The Equivalent Force Modeling (EFM) model from [19] is also carried out in order to have a comparison with the ESM approach and the direct piezoelectric coupling is also implemented in the EFM model. The structural model in EFM approach has 4307 nodes resulting in 21,535 degrees of freedom. In the ESM approach, each equivalent substructure model of the plate contains 17 nodes resulting in 51 degrees of freedom including both the transverse displacement and two rotational degrees of freedom. There are only 363 degrees of freedom in the equivalent system model which is much smaller than the full FE model. The order of the equivalent substructure model can be further reduced by reselecting the master nodes of the substructures. A proportional damping is adopted in both models:

$\boldsymbol{C}=\boldsymbol{\xi} \boldsymbol{M}+\eta \boldsymbol{K}$

where $\xi$ and $\eta$ are the proportional damping coefficients. As the proportional damping remains the orthogonality properties of the structural modes. (31) is rewritten as follows in modal coordinates [26]:

$\lambda_{i}=\frac{1}{2 \omega_{i}} \xi+\frac{\omega_{i}}{2} \eta$

where $\lambda_{i}$ and $\omega_{i}$ are the critical damping ratio and natural frequency of the $i^{\text {th }}$ mode. Hence, the damping coefficients can be estimated by using two experimental critical damping ratios of $0.27 \%$ and $0.15 \%$ for the modes at $39.87 \mathrm{~Hz}$ and $284.06 \mathrm{~Hz}$, respectively. The corresponding damping coefficients are given in Table 3.

\subsection{Modal parameter validation}

The natural frequencies validation is given in Table 2. One can observe that the equivalent system model agrees well with the full FE model and experimental data. The first five mode shapes of the studied plate are presented in Fig. 9. Although a limited number of master nodes are retained in the equivalent system model, the equivalent model can well describe the modes of the original system. Therefore the modal parameters well remain in the ESM model.

\subsection{Dynamic response validation}

Frequency response validations are also carried out and the validated frequency bandwidth is limited in $20 \mathrm{~Hz}-1000 \mathrm{~Hz}$. The FRFs input and output locations on the plate can be found in Fig. 6. The size of EFM model is reduced by dynamic condensation technique to reduce the computing time of the frequency responses, and many modeling information such as the placement of the transducers is lost after the model order reduction. The correction factor of shear-lag effect in the experiment due to the adhesive layer $\alpha_{p}=0.6808$ is estimated according to the work in [21] with an adhesive layer of $15 \mu \mathrm{m}$ thick. The electric field correction factor for MFC-d33 transducer $\alpha_{E}=0.8948$ is estimated by the solution in [20] and the dimensional characterization of the used MFC transducers [27]. These two corrections are included in the numerical simulations.

\subsubsection{Piezoelectric coupling validation}

The direct piezoelectric effect converts an mechanical input into an electric output. The piezoelectric induced mechanical properties caused by the capacitance of the transducer $C_{p}=1.15 n F$ and the external resistive load $R=1 M \Omega$ are considered in the numerical models. (30a) and (30b) can be rewritten as follows in frequency domain:

$$
\begin{aligned}
& \left(\boldsymbol{K}+j \omega \boldsymbol{C}-\omega^{2} \boldsymbol{M}\right) \boldsymbol{w}=\boldsymbol{\Theta}_{f} F+\boldsymbol{\Theta}_{w \phi} V, \\
& j \omega C_{p} V+\frac{V}{Z e}=j \omega \boldsymbol{\Theta}_{\phi w} \boldsymbol{w}
\end{aligned}
$$

where $F$ is an external force and $\Theta_{\mathrm{f}}$ is the force localization matrix. As the electric dynamics is also involved in the mechanical dynamics, it should be properly included. (33b) which describes the electric dynamics can be rewritten as:

$V=j \omega\left(j \omega C_{p}+\frac{1}{Z e}\right)^{-1}\left(\boldsymbol{\Theta}_{\phi w} \boldsymbol{w}\right)$.

Substituting (34) into (33a) yields the dynamics of the composite plate as follows:

$$
\begin{aligned}
& \left(\boldsymbol{K}+j \omega \boldsymbol{C}-\omega^{2} \boldsymbol{M}-j \omega\left(j \omega C_{p}+\frac{1}{Z e}\right)^{-1} \boldsymbol{\Theta}_{w \phi} \boldsymbol{\Theta}_{\phi w}\right) \boldsymbol{w} \\
& =\boldsymbol{\Theta}_{f} F .
\end{aligned}
$$

(33b) describes the voltage output of an integrated MFC transducer on the composite plate:

$V_{\text {out }}=\frac{j \omega}{j \omega C p+\frac{1}{Z_{e}}} \boldsymbol{\Theta}_{\phi w} \boldsymbol{w}$

where $\boldsymbol{w}$ depends on the dynamics of the system. (36) can be rewritten as follows by introducing (35):

$\frac{V_{\text {out }}}{F}=\frac{j \omega}{j \omega C_{p}+\frac{1}{Z e}} \boldsymbol{\Theta}_{\phi w} \frac{1}{\boldsymbol{K}+j \omega \boldsymbol{C}-\omega^{2} \boldsymbol{M}-j \omega\left(j \omega C_{p}+\frac{1}{Z e}\right)^{-1} \boldsymbol{\Theta}_{w \phi} \boldsymbol{\Theta}_{\phi w}} \boldsymbol{\Theta}_{f}$

The frequency response validations of the proposed piezoelectric coupling are presented in Fig. 10 and 11. The piezoelectric induced mechanical properties caused by the capacitance of the transducer $C_{p}=1.15 \mathrm{nF}$ and the external resistive load $R=1 \mathrm{M} \Omega$ are considered in the numerical models. It is shown in Fig. 10 and 11 that the numerical 
models agree well with the experimental measurement. The small mismatch of the FRFs' input and output placement between numerical models and tests and the material uncertainties of the composite plate as well as the MFC transducers contribute to the slight deviation of the FRFs. Besides, the bond-layer of the transducer also plays a rolebecause the transducers might be pre-stressed by the bond-layer in the experiment.

\subsubsection{Inverse piezoelectric coupling validation}

The inverse piezoelectric coupling transfers an electric input into a mechanical output on the composite plate. The dynamics of the system can be described as:

$$
\left(\boldsymbol{K}+j \omega \boldsymbol{C}-\omega^{2} \boldsymbol{M}\right) \boldsymbol{w}=\boldsymbol{\Theta}_{f} F+\boldsymbol{\Theta}_{w \phi} V,
$$

$$
j \omega C_{p} V-\frac{V}{Z e}=j \omega \boldsymbol{\Theta}_{\phi w} \boldsymbol{w} .
$$

In opposite with the direct piezoelectric effect, the voltage $V$ is an input of the inverse piezoelectric effect so that the term of the electric circuit has a minus sign. (38b) which describes the electric dynamics can be rewritten as:

$V=C_{p}^{-1}\left(\boldsymbol{\Theta}_{\phi w} \boldsymbol{w}+Q_{q}\right)$

where $Q_{q}$ is the charge due to the voltage input. Thus, the dynamics of the electric circuit is included in $Q_{q} . C_{p}^{-1} Q_{q}$ is actually the voltage $V$ which is applied to the transducer. Substituting (39) into (38a) yields the dynamics of the composite plate as follows:

$\left(\left(\boldsymbol{K}-\boldsymbol{\Theta}_{w \phi} C_{p}^{-1} \boldsymbol{\Theta}_{\phi w}\right)+j \omega \boldsymbol{C}-\omega^{2} \boldsymbol{M}\right) w=\boldsymbol{F}+\boldsymbol{\Theta}_{w \phi} V$

where $\boldsymbol{\Theta}_{w \phi} C_{p}^{-1} \boldsymbol{\Theta}_{\phi w}$ is the piezoelectric induced-stiffness caused by the capacitance of the transducer. Let $v$ to be the transverse velocity on the plate which is defined by the localization matrix $\boldsymbol{\Theta}_{v} \cdot v$ is expressed as follows in frequency domain:

$v=j \omega \boldsymbol{\Theta}_{v} \boldsymbol{w}$.

In piezoelectric transducer actuation case, there is no external force $F$. Substituting (40) into (41) yields:

$\frac{v}{V_{i n}}=\boldsymbol{\Theta}_{v} \frac{j \omega}{\left(\boldsymbol{K}-\boldsymbol{\Theta}_{w \phi} C_{p}^{-1} \boldsymbol{\Theta}_{\phi w}\right)+j \omega \boldsymbol{C}-\omega^{2} \boldsymbol{M}} \boldsymbol{\Theta}_{w \phi}$.

The frequency response validations of the inverse piezoelectric coupling are shown in Fig. 12 and 13. It can be observed that the ESM model agree well with both the EFM model and experimental data. The fluctuation of the experimental measurement at low frequency in Fig. 13 is mainly due to the small signal-to noise-ratio. The slight difference between the predicted FRFs and experimental data is mainly caused by the small error of the input and output placement in numerical models and tests. In addition, the material uncertainties of the composite plate and the MFC transducers also contribute to the FRFs' deviation.

\section{Study cases}

Two study cases are presented in this section to demonstrate the potential of the ESM approach. The energy harvesting one shows that the structure-preserving low-order model can be effectively used to design certain important parameters in dynamic applications. The piezoelectric shunted damping verified that the presented modeling approach can well simulate the induced mechanical effect due to the external electrical components.

\subsection{Energy harvesting}

The direct piezoelectric effect is widely studied for energy harvesting application. The performance of a MFC transducer is dominated by its piezoelectric fibrous orientation when its placement and external resistive load are fixed. It could be very difficult to optimize the piezoelectric fibrous orientation because it modifies both the mechanical and piezoelectric aspects. Especially, model order reduction is always required in practice for large-scale systems. The ESM approach can efficiently check the effect of the piezoelectric fibrous orientation on the electric output of the transducer. The voltage over force FRF for a set of piezoelectric fibrous orientation on the composite plate is shown in Fig. 14 and 15. The input of the FRFs is a concentrated force at $p 74$, and the output is the voltage extracted by the resistive load $R=1 M \Omega$ connected to the transducers.

The numerical FRFs for $\boldsymbol{\theta}=0^{\circ}$ agree well with the experimental data. One can observe from the two figures that the observability of each transducer depends on its piezoelectric fiber orientation. The MFC transducer at the corner of the composite plate captured more modes than the one at the center of the plate in low-frequency range. Given that the peak voltages usually are the most significant contributions in energy harvesting, it can be observed from the two figures that the optimal fiber orientation of the transducer at the center of the plate is in $\pm\left[0^{\circ}-15^{\circ}\right]$ by comparing the peak values. The optimal fiber orientation of the transducer at the corner is limited in $\pm\left[45^{\circ}-75^{\circ}\right]$. Nevertheless, it is worthwhile to mention that it is more difficult than this example to design the optimal piezoelectric fibrous orientation because the output of the transducers depends on the placement of transducers, external mechanical input as well as the design of the electrical device. The presented approach offers high flexibility for designing and modeling advanced energy harvesting system.

\subsection{Piezoelectric shunted damping}

A piezoelectric shunted damping example is used to demonstrate the presented modeling approach allows to include the dynamics of electrical components. Fig. 16 shows the piezoelectric shunted damping system that used in the verification.

Given that both the inductor $L$ and resistor $R$ are connected to the transducer in parallel, (33b) yields the following relation in frequency domain:

$V\left(j \omega C_{p}+\frac{1}{R}+\frac{1}{j \omega L}\right)-j \omega \boldsymbol{\Theta}_{\phi w} \boldsymbol{w}=0$.

Hence, the electric dynamics has a resonant frequency $\omega_{e}=\frac{1}{L C_{p}}$. Substituting (43) into (33a) yields:

$\left(\boldsymbol{K}+j \omega \boldsymbol{C}-\omega^{2} \boldsymbol{M}-\frac{j \omega \boldsymbol{\Theta}_{w \phi} \boldsymbol{\Theta}_{\phi w}}{j \omega C_{p}+\frac{1}{R}+\frac{1}{j \omega L}}\right) \boldsymbol{w}=\boldsymbol{\Theta}_{f} F$.

The velocity over disturbance FRF can be given as:

$\frac{v}{F}=\boldsymbol{\Theta}_{v} \frac{j \omega}{\boldsymbol{K}+j \omega \boldsymbol{C}-\omega^{2} \boldsymbol{M}-\frac{j \omega \boldsymbol{\Theta}_{w \phi} \boldsymbol{\Theta}_{\phi w}}{j \omega C_{p}+\frac{1}{R}+\frac{1}{j \omega L}}} \boldsymbol{\Theta}_{f}$.

When $\omega_{e}$ is close to s specific mode of the composite plate, the electric dynamics will resonants together with the mode, shunted damping can be introduced to that mode by the electric circuit. Hence, $L$ depends on the targeted mode because $C_{p}$ is fixed. and $R$ is the tuning parameter which dominates the shunted damping. From Fig. 14 and 15, one can observe that the transducer at the center of the plate can well capture the mode at $41 \mathrm{~Hz}$ and the one at the corner can well observe the modes at $283.5 \mathrm{~Hz}$. Therefore the two modes are targeted to be damped 
by the two transducers, respectively. Each transducer is connected by an individual electric circuit which is shown in Fig. 16. The corresponding inductance $L$ are $1221.1 \mathrm{H}$ and $269 \mathrm{H}$, respectively. The resistances $R$ are tuned as $1.3 \times 10^{8} \Omega$ and $2.5 \times 10^{7} \Omega$ for the two modes according to an engineering expertise. In fact, the optimal $R$ can be obtained by minimizing (45).

The velocity over force FRF is shown in Fig. 17. Although the used $L$ and $R$ might be not the optimal setting, the piezoelectric shunted damping achieved $8.46 \mathrm{~dB}$ and $12.56 \mathrm{~dB}$ amplitude reductions on the modes at $41 \mathrm{~Hz}$ and $283.5 \mathrm{~Hz}$, respectively. The performance of the shunted damping is quite good, given the quite limited size of the MFC transducer comparing to the composite plate. All the properties including the efficiency of the transducers and damping of the composite plate are included in the model. Therefore, the ESM model can simulate the effect induced by both the inductance and resistance. The optimization of the piezoelectric shunted damping solution could be analyzed, given that the low-order model significantly reduces the computing time.

\section{Conclusion}

Modeling of laminated composite plates with integrated rectangular MFC transducer is studied in this paper. An effective modeling approach is presented to generate structure preserving low-order system models for dynamic applications. The host composite plate and MFC transducers can be individually treated in the presented modeling approach. On the mechanical aspect, an equivalent substructure concept is used to generate the low-order structural model of the studied subject. On the piezoelectric aspect, the direct piezoelectric coupling of rectangular MFC transducers is derived from the electrical boundary conditions of the system and the corresponding inverse piezoelectric coupling is simulated by the equivalent forces. Therefore, the piezoelectric induced mechanical properties can be included in the system models.

A laminated composite plate with integrated MFC transducers is used for validations. The ESM approach is used to simulate the composite plate, and the experimental data is used to validate the numerical models. The modal parameter validation demonstrated the high fidelity of the structural models and frequency response validations showed that the presented low-order system model can well predict the dynamics of the piezoelectric system. Two dynamic application study cases are given to demonstrate the potential of the ESM approach. The energy harvesting one showed that specific parameters in the system model could be efficiently redefined for the optimal design of piezoelectric composite systems. The piezoelectric shunted damping case verified that the low-order system model can deal with the dynamics of the external electrical components.

The ESM approach offers high flexibility to design the integrated piezoelectric transducers on composite plate-type structures. The external electric component can also be considered into the system modeling. The generated low-order system models can be effectively used in practice in various dynamic applications such as energy harvesting and vibration suppression methodologies.

\section{Acknowledgment}

The Research Fund KU Leuven is gratefully acknowledged for its support. The European Commission is gratefully acknowledged for their support of the ARRAYCON research project (GA 605087).

\section{References}

[1] A. Ferreira, P. Novoa, A. Marques, Multifunctional material systems: a state-of-the-art review, Compos Struct 151 (2016) 3-35.

[2] Wikie W, Inman D, High J, Williams R. Recent developments in nasa piezocomposite actuator technology. In: 9th International Conference on New Actuators, Berman, Germany; 2004. 4p.

[3] X. Lin, K. Zhou, X. Zhang, D. Zhang, Development, modeling and application of piezoelectric fiber composites, Trans Nonferrous Metals Soc China 23 (2013) 98-107.

[4] S. Zhang, R. Schmidt, P. Muller, X. Qin, Disturbance rejection control for vibration suppression of smart beams and plates under high frequency excitation, J Sound Vib 353 (2015) 19-37.

[5] L. Gao, Q. Lu, F. Fei, L. Liu, Y. Liu, J. Leng, Active vibration control based on piezoelectric smart composite, Smart Mater Struct 22 (125032) (2013) 1-12.

[6] Steiger K, Mokry P. Finite element analysis of the macro fiber composite actuator: macroscopic elastic and piezoelectric properties and active control thereof by means of negative capacitance shunt circuit. Smart Mater Struct 24.

[7] Y. Chai, Z. Song, F. Li, Active aerothermoelastic flutter suppression of composite laminated panels with time-dependent boundaries, Compos Struct 179 (2017) 61-76.

[8] Y. Yang, L. Tang, H. Li, Vibration energy harvesting using macro-fiber composites, Smart Mater Struct 18 (2009), 8pp.

[9] Song H, Choi Y-H, Wereley N, Purekar A. Energy harvesting devices using macro-fiber composite materials. J Intell Mater Syst Struct 21.

[10] Ju S, Chae S, Choi Y, Ji C-H. Macro fiber composite-based low frequency vibration energy harvester. Sens Actuat A: Phys 226.

[11] S. Zhang, Y. Li, R. Schmidt, Modeling and simulation of macro-fiber composite layered smart structures, J Compos Struct 126 (2015) 89-100.

[12] S. Zhang, Z. Wang, X. Qin, G. Zhao, R. Schmidt, Geometrically nonlinear analysis of composite laminated structures with multiple macro-fiber composite (MFC) actuators, Compos Struct 130 (2016) 62-72.

[13] B. Nielsen, M. Nielsen, I. Santos, A layered shell containing patches of piezoelectric fibers and interdigitated electrodes: finite element modeling and experimental validation, Intell Mater Syst Struct 28 (2017) 78-96.

[14] D. Marinkovic, G. Rama, Co-rotational shell element for numerical analysis of laminated piezoelectric composite structures, Compos Part B 125 (2017) 144-156.

[15] Z. Bai, Y. Su, Dimension reduction of large-scale second-order dynamical systems via a second-order arnoldi method, Soc Ind Appl Math 26 (2005) 1692-1709.

[16] S. Gugercin, A. Antoulas, A survey of model reduction by balanced truncation and some new results, Int J Control 77 (2004) 748-766.

[17] J. Sullivan, J. Hubbard, S. Burke, Modeling approach for two-dimensional distributed transducers of arbitrary spatial distribution, J Acoust Soc Am 99 (1996) 2965-2974.

[18] A. Deraemaeker, G. Tondreau, Equivalent loads for two-dimensional distributed anisotropic piezoelectric transducers with arbitrary shapes attached to thin plate structures, J Acoust Soc Am (2011) 681-690.

[19] Dong Z, Faria C, Hromcik M, Pluymers B, Sebek M, Desmet M. Equivalent force modeling of macro fiber composite actuators integrated into non-homogeneous composite plates for dynamic applications. Smart Mater Struct 26.

[20] H. Nasser, A. Deraemaeker, S. Belouettar, Electric field distribution in macro fiber composite using interdigitated electrodes, Adv Mater Res 47-50 (2008) 1173-1176.

[21] Dan C, Malinowski P, Kudela P, Opoka S, Radzienski M, Mieloszyk W, et al. Validation of macro fiber composites for strain measurements in structural health monitoring applications of complex aerospace structures. In: Proceedings of the 9th International Conference on Structural Dynamics, EURODYN, Porto, Portugal.

[22] Z.-Q. Qu, R. Selvam, Dynamic superelement modeling method for compound dynamic systems, AIAA J 38 (2000) 1078-1083.

[23] Y. Li, S. Zhang, R. Schmidt, X. Qin, Homogenization for macro-fiber composites using reissner-mindlin plate theory, J Intell Mater Syst Struct 27 (2016) 2477-2488.

[24] R. Williams, Nonlinear mechanical and actuation characterization of piezoceramic fiber composites, [Ph.D. thesis] Virginia Polytechnic Institute and State University, 2004.

[25] K. Beltzinger, M. Bischoff, E. Ramm, A unified approach for shear-locking free triangular and rectangular shell finite elements, Comput Struct 75 (2000) 321-334.

[26] E. Wilson, Static and dynamic analysis of structures, 4th ed., Computers and Structures Inc, Berkeley, CA, 2004.

[27] S. Hensel, W. Drossel, M. Nestler, R. Muller, Modeling of the performance reduction of macro fiber composites for use in numerical forming simulation of piezoceramic-metal-compounds, CIRP J Manuf Sci Technol 7 (2014) 129-138.

[28] Smart Material Corp. Macro fiber composite-MFC,www.smart-material.com, [accessed on 2018-02-12]. 